\title{
The Coherence Time of polar slab
}

\begin{tabular}{|r|l|}
\hline Journal: & Canadian Journal of Physics \\
\hline Manuscript ID & cjp-2017-0272.R1 \\
\hline Manuscript Type: & Article \\
\hline Date Submitted by the Author: & 15-Jul-2017 \\
\hline Complete List of Authors: & wang, xiuqing; Inner Mongolia National University \\
\hline $\begin{array}{r}\text { Keyword: } \\
\text { Is the invited manuscript for } \\
\text { consideration in a Special } \\
\text { Issue? : }\end{array}$ & $\begin{array}{l}\text { polar slab, Coherence time, Pekar type variational method, coupling } \\
\text { constant, slab thickness }\end{array}$ \\
\hline
\end{tabular}




\title{
The Coherence Time of polar slab
}

\author{
Xiu-qing WANG ${ }^{1}$ \\ College of Physics and Electromechanics, Inner Mongolia National University, \\ Tongliao 028043, China
}

\begin{abstract}
The relationship among the Coherence time (CT) $\tau$, Variation frequency $\lambda$, slab thickness $d$, energy separation $\Delta E$ and coupling constant $\alpha$ in polar slab was investigated using Pekar type variational method. The results indicated that the $\tau$ is positively proportional to the $\lambda$, but the $\Delta E, d$ and $\mathrm{t} \alpha$ are negatively correlated with the $\tau$. When $\Delta E$ is more than $10 \mathrm{ev}$, and $d$ is less than $5 \mathrm{~nm}, \tau$ decreases sharply.
\end{abstract}

Key words: polar slab; Coherence time (CT); Pekar type variational method

\section{Introduction}

With the fast development of the epitaxial technique and the manufacturing of semiconductor materials in recent years, considerable interest has been focused on the investigation of the polar slab, quantum well and semiconductor superlattice because of their potential application in the quantum computation (QC) process. However, in order to perform QC, two crucial issues of the basic conditions should be solved: (i) a stable two-level system, which can be envisagedas a single qubit; (ii) a sufficiently long $\mathrm{CT}$ to ensure the implementation of $\mathrm{QC}^{[1]}$. In view of these, the $\mathrm{CT}$ of qubit should be prolonged or increased, which is the subject of the present paper.

In fact, such a topic has been addressed very recently. Petta et $\mathrm{a}^{[2]}$ have demonstrated a coherent control of a quantum two-level system based on two-electron spin states and observed a CT for two-electron spin states exceeding 1 microsecond in a double quantum dots (QDs), allowing state preparation, coherent manipulation, and projective readout. Kaji et al ${ }^{[3]}$ have investigated and discussed in detail the effects of valence band mixing on the hole spin coherence in self-assembled InAlAs QDs. Varwig et al ${ }^{[4]}$ have studied the temperature dependence of the CT of hole spins confined in self-assembled (In,Ga)As/GaAs QDs by spin-mode-locking and spin-echo techniques, suggesting that the spin-orbit-related interactions were the decisive sources for hole spin decoherence. Persistent quantum coherence between the $1 \mathrm{~S}$ and $1 \mathrm{P}$ excitonic states at room temperature were reported using two-dimensional electronic spectroscopy by Caram et al ${ }^{[5]}$ in zinc-blende colloidal CdSe QDs, suggesting an avenue for engineering long-lived interexcitonic quantum coherence. Dependences of particle size and magnetic field on the electron spin coherences in colloidal CdSe QDs with zinc blende structure are investigated using ultra fast time-resolved Faraday ellipticity at room temperature by Zhang et al ${ }^{[6]}$. Actually, in most of the above-mentioned studies, the phenomena of coherence are considered in the QD. Coherence time in a polar slab, however, has not been studied so far by the Pekar type variational method.

Foundation item: Project Supported by Inner Mongolia University for the Nationalities (NJZY174) E-mail address: wxq_1975@163.com 
In this paper, we have investigated the effects of Coherence Time $\tau$, the energy separation $\Delta E$ and coupling constant $\alpha$ in a parabolic quantum well by using Pekar type variational method.

\section{Theoretical Model}

We used the system of electron-bulk LO phonon coupling strong, Therefore, the Hamiltonian of the system can be written as ${ }^{[7]}$

$$
\begin{aligned}
& H=H_{e}+H_{p h}+H_{e-p h} \\
& H_{e}=\frac{p_{z}^{2}}{2 m_{b}}+\frac{\hbar^{2} k_{\rho}^{2}}{2 m_{b}}+V(z)
\end{aligned}
$$

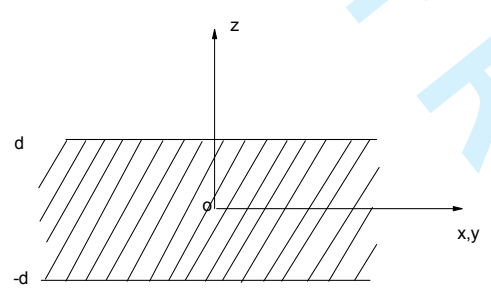

Fig.1 Geometry of semiconductor

slab

$$
\begin{aligned}
& V(z)=\left\{\begin{array}{l}
0,|z| \leq d \\
\infty,|z|>d
\end{array}\right. \\
& H_{e-p h}=H_{e-S O}+H_{e-L O}
\end{aligned}
$$

$\alpha=\frac{e^{2}}{2 \hbar \omega_{L O}}\left(\frac{2 m_{b} \omega_{L O}}{\hbar}\right)^{\frac{1}{2}}\left(\frac{1}{\varepsilon_{\infty}}-\frac{1}{\varepsilon_{0}}\right)$ is the electron- phonon coupling constant.

All the means of physical volume are the same as that described in $\operatorname{Ref}^{[7]}$

For the convenience of calculation we took the value $\hbar=2 m_{b}=1$, With

$$
U=\exp \left\{\sum_{m, \mathrm{k}}\left[f_{m} a_{m}^{+}(\vec{k})+f_{m}^{*} a_{m}(\vec{k})\right]+\sum_{q, t}\left[f_{t} b_{t}^{+}(\vec{q})-f_{t}^{*} b_{t}(\vec{q})\right]\right\}
$$

Here $f_{m}, f_{t}\left(f_{m}^{*}, f_{t}^{*}\right)$ stands for variational function that will be subsequently chosen by 
minimizing the energy.

$$
\mathrm{H}^{\prime}=\mathrm{U}^{-1} H U
$$

Under the Gauss function approximation following the Pekar type variational method the trial wavefunction can be written as ${ }^{[8]}$

$$
\begin{aligned}
& \left|\varphi_{\mathrm{e}-\mathrm{p}}\right\rangle=\frac{\lambda}{\sqrt{\pi}} \exp \left(-\frac{\lambda^{2} \rho^{2}}{2}\right)|\xi(z)\rangle\left|0_{p h}\right\rangle \\
& \langle\xi(z) \| \xi(z)\rangle=\delta(z),\left|0_{p h}\right\rangle \text { is Zero phonon state, satisfied } a_{m, t}(k)\left|0_{p h}\right\rangle=0 \text {, then the }
\end{aligned}
$$

ground state energy of electrons is

$$
E_{0}(\lambda)=\left\langle\varphi_{e-p}\left|H^{\prime}\right| \varphi_{e-p}\right\rangle
$$

$$
E_{0}=\lambda^{2}-\frac{\alpha \sqrt{\omega_{L O}}}{\pi^{2}}\left(\sum_{m=1,3,5 \ldots} \cos ^{2} \frac{m \pi z}{2 d}+\sum_{m=2,4,6 \ldots} \sin ^{2} \frac{m \pi z}{2 d}\right)\left[\frac{\sqrt{\pi}}{2} \frac{1}{\lambda}-\frac{1}{\lambda^{2}} \int_{0}^{\infty} \frac{\left(\frac{m \pi}{2 d}\right)^{2}}{\mathrm{q}^{2}+\left(\frac{m \pi}{2 d}\right)^{2}} e^{-\frac{q^{2}}{2 \lambda^{2}}} d q\right]
$$

The trial wavefunction of the first excited states can be written as

$$
\left|\varphi_{e-p}\right\rangle^{\prime}=\frac{\lambda^{2}}{\sqrt{\pi}} \rho \exp \left(-\frac{\lambda^{2} \rho^{2}}{2}\right) \exp ( \pm \mathrm{i} \varphi)|\xi(z)\rangle\left|0_{p h}\right\rangle
$$

satisfied

$$
\left\langle\varphi_{e-p} \mid \varphi_{e-p}\right\rangle^{\prime}=0,\left\langle\varphi_{e-p} \mid \varphi_{e-p}\right\rangle^{\prime}=1
$$

The first excited state energy of the electron can be obtained from the formula

$$
E_{1}=\left\langle\varphi_{e-p}\left|H^{\prime}\right| \varphi_{e-p}\right\rangle^{\prime}
$$

then

$$
E_{1}=\lambda^{2}-\frac{2 \alpha \sqrt{\omega_{L O}}}{\pi^{2}}\left(\sum_{m=1,3, \ldots} \cos ^{2} \frac{m \pi z}{2 d}+\sum_{m=2,4,6 \ldots} \sin ^{2} \frac{m \pi z}{2 d}\right)\left\{\begin{array}{l}
\frac{3 \sqrt{2 \pi}}{4} \frac{1}{\lambda}-\frac{2}{\lambda^{2}} \int_{0}^{\infty} \frac{\left(\frac{m \pi}{2 d}\right)^{2}}{\mathrm{q}^{2}+\left(\frac{m \pi}{2 d}\right)^{2}} e^{-\frac{q^{2}}{2 \lambda^{2}}} d q \\
+\frac{1}{2 \lambda^{4}} \int_{0}^{\infty} \frac{\mathrm{q}^{2}\left(\frac{m \pi}{2 d}\right)^{2}}{\mathrm{q}^{2}+\left(\frac{m \pi}{2 d}\right)^{2}} e^{-\frac{q^{2}}{2 \lambda^{2}}} d q
\end{array}\right\}
$$


We could obtain $\lambda_{0}$ using variational method and the eigen levels and eigen wavefunctions were gotten. So a two-level system as a single qubit was built up. superposition state of electron in a quantum well could be expressed as

$\left|\psi_{01}\right\rangle=\frac{1}{\sqrt{2}}(|0\rangle+|1\rangle)$

$\omega_{01}=\frac{\left(E_{1}-E_{0}\right)}{\hbar}$ is electron transition frequency

Under the dipole approximation, based on the Fermi Golden Rule, the spontaneous emission rate can be written as ${ }^{[9]}$

$$
\tau^{-1}=\frac{e^{2} \Delta E}{3 \pi \varepsilon_{0} \hbar^{2} m_{b}^{2} c^{3}} \sqrt{\frac{\varepsilon}{\varepsilon_{0}}}|\langle 0|\rho| 1\rangle|^{2}=\frac{3 e^{2} \Delta E}{4 \lambda_{0}^{4} \pi \varepsilon_{0} \hbar^{2} m_{b}^{2} c^{3}} \sqrt{\frac{\varepsilon}{\varepsilon_{0}}}
$$

Where $C$ is the speed of light in vacuum, $\varepsilon\left(\varepsilon_{0}\right)$ is the material (vacuum) dielectric constant,

$\Delta E=E_{1}-E_{0}$ is the energy separation, $\tau$ is the Coherence time.

\section{Numerical results and discussion}

Subsequently, the numerical calculations have been carried out to determine the effects of the frequency $\lambda$, the slab thickness, the energy separation $\Delta E$ and coupling constant $\alpha$ in polar slab.

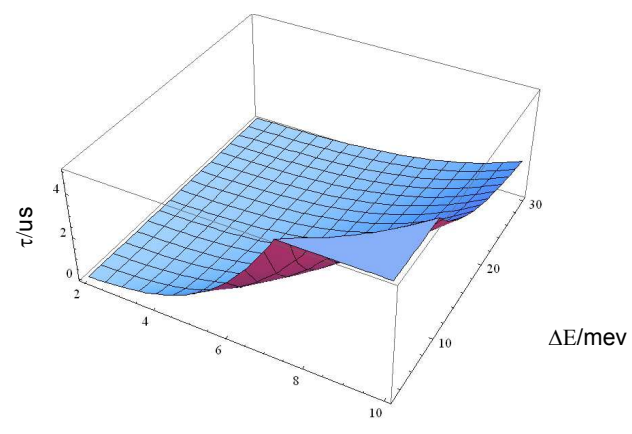

$\lambda / 10^{12} \mathrm{HZ}$ 


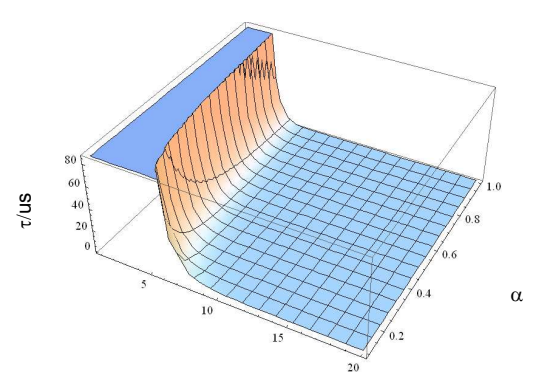

$\mathrm{d} / \mathrm{nm}$

Fig. 3 The relationship among the $\tau$, the $d$ and the $\alpha(\alpha \leq 1)$

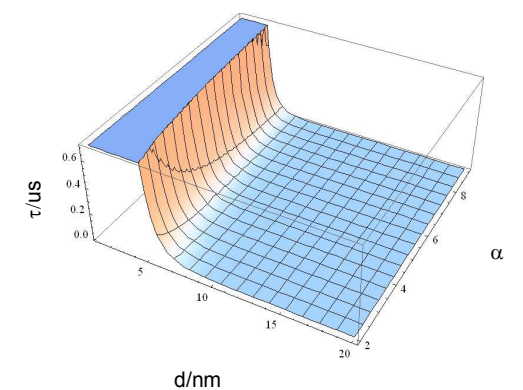

Fig. 4 The relationship among the $\tau$, the $d$ and the $\alpha(\alpha>1)$

Fig. 2 showed the relationship among the Coherence time $\tau$, the Variation frequency $\lambda$ and the energy separation $\Delta E$. The results demonstrated that $\tau$ increases with $\lambda$ but decreases with $\Delta E$. When $\Delta E>10 e v, \tau$ was reduced notable.

Fig. 3 and Fig. 4 showed The relationship between the Coherence time $\tau$, the slab thickness $d$ and coupling constant $\alpha$. It can be seen that $\tau$ is a decreasing function of $d$ and $\alpha$. And When $d<5 \mathrm{~nm}, \tau$ was reduced sharply.

As shown in figures 2, 3 and 4, the Coherence time $\tau$ increased with the increase in Variation frequency $\lambda$. This is due to the fact that the smaller the Variation frequency is, the higher the energy separation is. but $\omega_{01}$ is enhanced with the increased $\Delta E$. In practice, qubits can't be separated from the environment and exist independently. With the increase of $\omega_{01}$, they must Interacted with environmental heat reservoirs, This resulted in the destruction of qubit superposition, which is decoherence. So $\tau$ increased with $\lambda$, but decreases with $\Delta E$. We can 
also see that $\tau$ is a decreasing function of coupling constant $\alpha$, the reason is that the stronger the electron-bulk LO phonon coupling is, the higher the $\Delta E$ is. $\omega_{01}$ is enhanced with the increased $\Delta E$. With the increase of $\omega_{01}$, to qubit superposition failure, which is decoherence. So $\tau$ is a decreasing function of coupling constant $\alpha$. Coherence time $\tau$ decreases with slab thickness $d$ When $d<5 \mathrm{~nm}$, since the narrower slab thickness $\mathrm{d}$ is, the stronger the electron-phonon interaction would be. These can also be attributed to the interesting quantum size confining effect.

\section{Conclusions}

The relationship among the Coherence time $\tau$, the slab thickness $d$, the Variation frequency $\lambda$, the energy separation $\Delta E$ and coupling constant $\alpha$ in polar slab was investigated using Pekar type variational method. The results indicated that the $\tau$ is positively proportional to the $\lambda$, but the energy separation $\Delta E$, slab thickness $d$ and coupling constant $\alpha$ are negatively correlated with the Coherence time $\tau$.

References:

[1] Li, S.S., Xia, J.B., Liu, J.L., Yang, F.H., Niu, Z.C., Feng, S.L., Zheng, H.Z.: Inas/gaas single-electron quantum dot qubit. J. Appl. Phys. 90, 6151-6155 (2001)

[2] Petta, J.R., Johnson, A.C., Taylor, J.M., Laird, E.A., Yacoby, A., Lukin, M.D., Marcus, C.M., Hanson, M.P., Gossard, A.C.: Coherent manipulation of coupled electron spins in semiconductor quantum dots. Science 309, 2180-2184 (2005)

[3]Kaji, R., Ohno, S., Hozumi, T., Adachi, S.: Effects of valence band mixing on hole spin coherence via hole-nuclei hyperfine interaction in inalas quantum dots. J. Appl. Phys. 113, 203511-6 (2013)

[4] Varwig, S., Rene, A., Greilich, A., Yakovlev, D.R., Reuter, D., Wieck, A.D., Bayer, M.: Temperature dependence of hole spin coherence in (in, ga) as quantum dots measured by mode-locking and echo techniques. Phys. Rev. B 87, 115307-6 (2013)

[5] Caram, J.R., Zheng, H., Dahlberg, P.D., Rolczynski, B.S., Griffin, G.B., Fidler, A.F.,

Dolzhnikov, D.S., Talapin, D.V., Engel, G.S.: Persistent interexcitonic quantum coherence in cdse quantum dots. J. Phys.Chem. Lett. 5, 196-204 (2014)

[6]Zhang, Z.B., Jin, Z.M., Ma, H., Xu, Y., Lin, X., Ma, G.H., Sun, X.L.: Room-temperature spin coherence in zinc blende cdse quantum dots studied by time-resolved faraday ellipticity. Physica. E 56, 85-89 (2014)

[7] Wang X Q, Self-trapping energy of bound magnetopolaron in a polar slab of semiconductor. J. Chin.J.Quantum Electronics, 27(2), 242-246(2010)

[8] Wang Zi-Wu, XIAO Jing_Lin, Parebolic linear bound potential quantum dot qubit and its optical phonon effect. J.Acta Physica Sinica, 56(02):678-681(2007)

[9] L. D. Landau, E. M. Lifshitz, Quantum Mechanics (Nonrelativistic Theory), London. (1987) 